\title{
Spinal Cord Segments Containing Key Elements of the Central Pattern Generators for Three Forms of Scratch Reflex in the Turtle
}

\author{
Lawrence I. Mortin and Paul S. G. Stein \\ Biology Department, Washington University, St. Louis, Missouri 63130
}

The immobilized, low-spinal turtle produces 3 forms of the fictive scratch reflex in response to tactile stimulation of specific sites on its body surface (Robertson et al., 1985). We used complete transections of the spinal cord at different rostrocaudal levels to reveal the minimum length of spinal cord sufficient to produce each scratch form. Additional transections revealed the progressive loss of elements of the motor pattern and the eventual loss of rhythmogenesis. We have identified, therefore, spinal cord segments containing key elements of each scratch form's central pattern generator (CPG).

The turtle spinal cord consists of 8 cervical segments (C1C8), 10 dorsal segments (D1-D10), 2 sacral segments (S1, S2) and about 16 caudal segments (Ca1-Ca16; Kusuma et al., 1979). The cell bodies of motor neurons innervating the hindlimb muscles are located in the hindlimb enlargement, segments D8-S2 (Ruigrok and Crowe, 1984). The receptive field for the rostral scratch is innervated by segments D3D6; the pocket scratch receptive field is innervated by segments D6-D8; the caudal scratch receptive field is innervated by segments $\mathrm{S2}, \mathrm{Ca1}$, and more caudal segments (Mortin and Stein, 1985).

A rostral scratch motor pattern could be produced with as few as 5 or 6 segments, i.e., segments D5-D9 or D3-D8. The anterior 3 segments of the hindlimb enlargement, D8-D10, could produce a pocket scratch motor pattern. A single segment, either $D 7$ or D8, is capable of rhythmogenesis in response to stimulation of sites in its part of the pocket receptive field. A caudal scratch motor pattern could be produced by D8-End (the hindlimb enlargement and more caudal segments). The posterior $40-80 \%$ of the hiridlimb enlargement is not necessary for the production of a rostral or pocket motor pattern. The anterior segment of the enlargement is necessary for the production of a normal caudal scratch motor pattern. Key elements of the CPG for each of the 3 scratch forms reside in segments D7-D10. The patterngenerating capacity of the anterior half of the hindlimb enlargement is greater than the posterior half; such an asymmetric distribution of pattern-generating elements in the enlargement of the spinal cord has been described for cat scratching (Deliagina et al., 1983). These results are con-

\footnotetext{
Received May 2, 1988; revised Dec. 20, 1988; accepted Dec. 22, 1988.

We wish to thank Gail A. Robertson for helpful discussions throughout this project and for critically reviewing the manuscript. This work was supported by NIH Grant NS15049 to P.S.G.S.

Correspondence should be addressed to Lawrence I. Mortin, Biology Department, Brandeis University, Waltham, MA 02254.

Copyright (C) 1989 Society for Neuroscience $0270-6474 / 89 / 072285-12 \$ 02.00 / 0$
}

sistent with the hypothesis that the CPGs producing different motor patterns for the hindlimb share neuronal elements (Grillner, 1981; Robertson et al., 1985; Currie and Stein, 1988, 1989).

A wide variety of rhythmical behaviors in vertebrates, such as swimming, walking, and scratching, are produced within the CNS by central pattern generators (CPGs; for reviews, see Grillner, 1981; Stein, 1984). A CPG is defined as the set of neurons within the CNS that, in the absence of movement-related sensory feedback, can produce the appropriate sequence of neural activities that underlie a behavior. Sensory feedback is essential for the adaptive functioning of these CPGs in an animal's natural environment. The CPGs underlying several different vertebrate behaviors reside within the spinal cord; these have been identified as spinal CPGs by recording the appropriate motor output after removing both phasic sensory feedback from the periphery and supraspinal influences (Stein, 1984). Which spinal cord segments contain neurons involved in pattern generation? The answer to this will help direct future experimental work with singlecell recording techniques designed to identify the neurons and circuits responsible for vertebrate pattern generation.

The turtle spinal cord contains a sensorimotor translator that produces 3 forms of the scratch reflex (Mortin et al., 1985; Robertson et al., 1985). Each form of the scratch exhibits a stereotypical motor output that directs the limb to rub against a set of receptive field sites covering a specific portion of the turtle's body. The 3 forms of scratch in the turtle are produced by CPGs within the spinal cord (Robertson et al., 1985). Each scratch form can be distinguished from the other 2 forms by the timing and intensity characteristics of their motor patterns. For each form of the scratch, hip protractor (VP-HP) motor activity rhythmically alternates with hip retractor (HR-KF) motor activity. Distinct for each form is the timing of activation of the monoarticular knee extensor (FT-KE) motor output in the activation cycle of the hip musculature. FT-KE is active in a rostral scratch during the latter portion of VP-HP activation; FT-KE is active in a pocket scratch during HR-KF activation; FT-KE is active in a caudal scratch after HR-KF activation has already occurred (Robertson et al., 1985).

This paper reports the identification of those spinal cord segments that contain key elements of the CPG for each scratch form. We activated scratch motor output using natural stimulation within each scratch form's receptive field. We monitored rhythmic hindlimb and respiratory motor output activated during each scratch form. We determined those spinal cord segments that were sufficient and some that were necessary for the production of each scratch form's motor pattern. Our technique had a major limitation: for each scratch form, we needed at 
least one segment of that form's receptive field connected with at least one segment that contained motor neurons active during that form. We could not determine whether or not additional elements of the rostral and pocket scratch generators are located in posterior enlargement segments. Our results are consistent with the hypothesis that there is sharing of neuronal elements involved in generating the 3 scratch forms. Portions of this work were described previously in an abstract (Mortin and Stein, 1985) and in a doctoral dissertation (Mortin, 1987).

\section{Materials and Methods}

Adult turtles, Pseudemys scripta elegans ( $n=34$; carapace length, 10$20 \mathrm{~cm}$; weight, $200-800 \mathrm{gm}$ ) were used for this study. Each turtle was placed in crushed ice for a minimum of $1 \mathrm{hr}$ prior to surgery to obtain hypothermic anesthesia (Melby and Altman, 1974; Maxwell, 1979; Mortin et al., 1985). Every turtle in this study was first spinalized midway between spinal cord segments dorsal 2 and dorsal 3 (D2 and D3); D2 is the second of 10 dorsal (postcervical and presacral) spinal segments (Mortin and Stcin, 1985; Mortin et al., 1985). All further surgical procedures were performed while the turtle was maintained in crushed ice, with the exception of the secondary spinal cord transections used to localize CPG elements of the scratch; these transections were performed at room temperature at the physiology set-up.

Peripheral nerves were surgically dissected from the hindlimb for electroneurographic (ENG) recordings (Robertson et al., 1985; Mortin, 1987). ENG recordings were obtained from 3 distinct regions: (1) the femoral nerve plexus of the hindlimb, including the nerves to VP-HP (puboischiofemoralis internus, pars anteroventralis, a hip protractor and femoral rotator muscle), IT-KE (triceps femoris, pars iliotibialis, a knee extensor, hip protractor, and hip abductor muscle), AM-KE (triceps femoris, pars ambiens, a knee extensor and hip adductor muscle), and FT-KE (triceps femoris, pars femorotibialis, a pure knee extensor musclc), and the distal D8 (dD8) nerve (innervating respiratory muscles and other muscles of the pelvic girdle); (2) the sciatic nerve plexus of the hindlimb, including the HR-KF nerve (innervating flexor cruris, pars flexor tibialis internus and several other muscles that retract the hip and flex the knee, and one muscle that adducts the hip); and (3) peripheral nerves from pre-enlargement segments D6 and D7 innervating respiratory muscles (Bojanus, 1819; Walker, 1973). The recording from each nerve was obtained via a pair of silver-wire electrodes in a pool of mineral oil. The ENG signals were amplified (100-1000 $\mathrm{Hz}$ bandpass) and stored on FM magnetic tape for later analysis and filming.

In addition to dissecting the peripheral motor nerves from one hindlimb, a long length of the spinal cord was exposed from the dorsal aspect (Mortin and Stein, 1985; Mortin, 1987). After removal from the ice, the turtle was allowed to warm to room temperature, and then immobilized with gallamine, a neuromuscular blocking agent, at a dosage of $6 \mathrm{mg} / \mathrm{kg}$ body weight. Scratch episodes, monitored with ENG recordings, were elicited by gentle mechanical stimulation of specific sites on the body surface at regular intervals throughout an expcriment (see Mortin et al., 1985, for the names of stimulus sites on the shell). The spinal cord was transected completely, midway between adjacent dorsal roots at different levels, with a single cut using fine iridectomy scissors. The motor pattern produced in response to scratch stimulation was compared before and after each transection. Spinal shock is minimal in the turtle, as motor patterns could be elicited within a few minutes after each transection. If a scratch motor pattern could be elicited, then the spinal cord piece left intact was sufficient to produce that fictive scratch form. Changes in the motor output after a transection indicated that either (1) a key CPG element residing in the deleted segment(s) was lost, (2) motor neurons that contributed to the motor output had somata in the deleted segment(s), or (3) that enabling elements, e.g., primary afferents or cutaneous sensory interneurons, for that scratch form's CPG were removed.

For each form of the scratch, the cycle period was measured from a number of representative scratch episodes in each reduced preparation. The cycle period was defined as the time from the onset or offset of activity in one motor nerve to the next occurrence of that same activity. Most often, cycle period was measured as the time from the onset of activity in one burst of the VP-HP nerve to the onset of VP-HP activity in the subsequent cycle. When the VP-HP nerve monitor was not available, the nerve displaying the most regular and robust activation during each cycle was utilized.

\section{Results}

\section{Rostral scratch generation}

The fictive rostral scratch motor pattern is characterized by the activation of the pure knee extensor nerve, FT-KE, during the latter half of the activation of the hip protractor nerve, VP-HP (Fig. 1A). Activation of VP-HP alternates with activation of the hip retractor nerve, HR-KF. VP-HP activation usually has a longer duration than HR-KF activation in the fictive rostral scratch. The extreme of this difference in duration between the VP-HP and HR-KF bursts is a natural variation, termed an $H R-K F$ deletion, seen during some rostral scratch episodes, in which the phase of activation of HR-KF is deleted and a second VP-HP burst occurs without an intervening HR-KF burst (formerly called a B-phase deletion by Stein and Grossman, 1980; and Stein et al., 1982; see also Stein, 1985; Robertson and Stein, 1988). The rostral scratch is further characterized by the sequential activation of IT-KE (near the offset of HR-KF, e.g., Fig. $1 B$ ), AM-KE, and then FT-KE, respectively. AM-KE, FT$\mathrm{KE}$, and VP-HP terminate their activity near the onset of HRKF.

Figure 1, a composite of scratch episodes from several experiments, shows that the posterior 2-4 segments of the 5-segment hindlimb enlargement are not necessary for the production of a rostral scratch motor pattern. Figure $1 A$ shows a control fictive rostral motor pattern, initiated by stimulation of a site in the rostral receptive field, from an immobilized turtle spinalized between segments D2 and D3. The last trace in Figure $1 A$ is a recording from the dD8 nerve, containing motor axons that innervate several muscles adjacent to the hindlimb, including 2 respiratory muscles. During the rostral scratch, the dD8 nerve showed 2 bursts of activity, one coactive with VP$\mathrm{HP}$ and the second coactive with HR-KF. The motor neurons whose axons project in the dD8 nerve are located mainly in segment D8. Thus, this nerve provides a monitor of the HRKF phase of the rostral scratch that, in contrast to the HR-KF motor pool itself, is located in the most anterior segment of the enlargement.

A preparation containing segments D3-D10 still produced the nerve synergies of the rostral motor pattern (Fig. 1B). In Figure $1 B$, FT-KE was active during the latter half of VP-HP activity, and both of these nerves were quiescent during HRKF activity. Other features that characterize the rostral scratch also remained unchanged compared with the control. Variations in the timing of IT-KE activity and the difference in cycle period are within the normal variability of rostral scratch episodes (cf. Fig. 1, $A, B$ ). A preparation containing segments D3-D9 produced a rostral motor pattern (Fig. $1 C$; the increased cycle period is discussed later). The sequence of activation of these nerves was the same as in the control. Thus, the posterior $60 \%$ of the enlargement, i.e., D10, S1, and S2, and all the caudal segments are unnecessary for the production of a rostral motor pattern.

A D3-D8 preparation generated a nearly normal rostral motor pattern (Fig. 1, D,E). At this stage, the preparation had only 1 of the 5 enlargement segments (D8), and therefore only a limited number of intact motor pools. Figure $1, D, E$, shows the motor patterns elicited by stimulation within the rostral receptive field of such a 6-segment preparation, in 2 different turtles. In Figure $1 D$, the timing of activation of the first 4 motor nerves was very similar to that seen in the control. There was no activity in HR$\mathrm{KF}$, the last trace in $D$; its motor pool is located mainly in segments D10, S1, and S2 (Ruigrok and Crowe, 1984), which 


\section{A. D3-End}

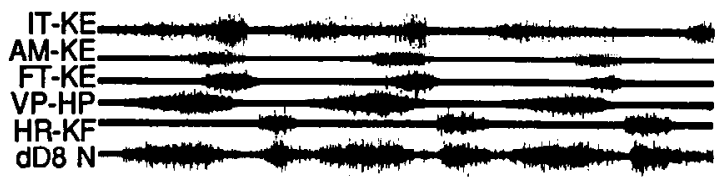

\section{B. D3-D10}

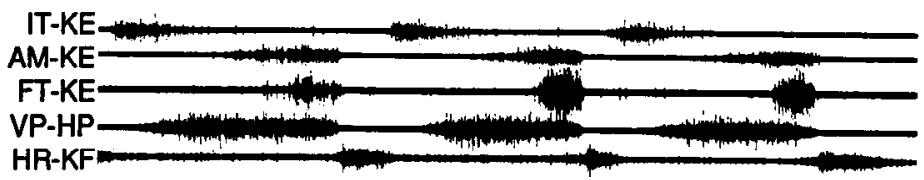

\section{D3-D9}

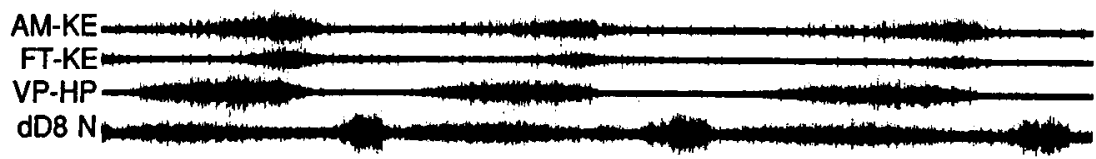

2 sec

\section{D3-D8}

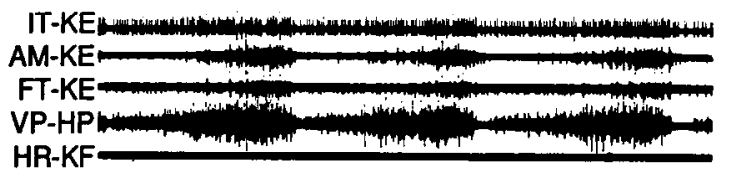

\section{E. D3-D8}

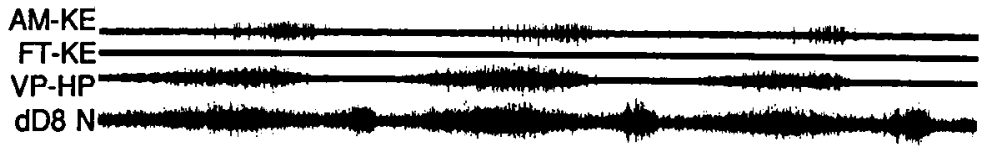

Figure 1. The posterior $40-80 \%$ of the hindlimb enlargement is not necessary for the production of a rostral scratch motor pattern. The spinal cord segments that were intact for each preparation are labeled above each motor response in this and subsequent figures. Each episode shows 3 cycles of a rostral motor pattern recorded in immobilized turtles. Six motor nerves were recorded in these preparations (see Materials and Methods). The dD8 $\mathrm{N}$ recording showed 2 distinct bursts in the rostral scratch, one coactive with VP-HP and the second coactive with HR-KF; it is likely that these represent separate motor pools. The stimulus positions that elicited each scratch response are as follows: $A, \mathrm{M} 8.0 ; B, \mathrm{SP} 1 ; C, \mathrm{SP} 3.5$; $D$, SP 3; and $E$, a site ventral to SP 2.

have been removed in this preparation. Each successive burst of activity in VP-HP began at or near the offset of the previous VP-HP burst, indicating a deletion of the HR-KF phase. HRKF deletions occurred with greater frequency after the removal of first segment D10, and then D9 (Fig. 2). Figure 2 shows the percentage of cycles that displayed clear hip-retractor phases in each of 5 different preparations in one turtle. The trend outlined in this experiment was a general finding in all rostral scratch experiments. The loss of segment Dl0, and even more so D9, resulted in a significant decrease in the probability of occurrences of the hip-retractor phase of the rostral scratch. Key CPG elements producing the hip-retractor phase of the rostral scratch are located in segments D9 and D10.

In Figure $1 E$, there were 2 bursts in the dD8 nerve for every burst in the VP-HP nerve; one was coactive with VP-HP, while the second, larger-amplitude burst was in antiphase with VP-HP activity. This second burst in the $\mathrm{dD} 8$ nerve demonstrates that, in some cases, a hip-retractor-correlated phase of nerve activity can occur in a D3-D8 preparation. There was no activity in the FT-KE nerve in this D3-D8 preparation (Fig. $1 E$ ). The turtle in $D$ still showed some FT-KE nerve activity in the absence of segment D9, although this activity was reduced in amplitude from the control. The FT-KE motor pool most often is located in segment D9. Occasionally some FT-KE units are located in D8. In the majority of our experiments, there was no FT-KE activity when D9 was absent (Fig. $1 E$ ). Taken together, Figure $1, D, E$, indicates that a motor pattern showing many of the synergies of a rostral scratch can be produced in a D3-D8 preparation. Overall, in 6 out of 11 turtles segments D3-D8 showed nerve activity synergies unique to the rostral motor pattern. In

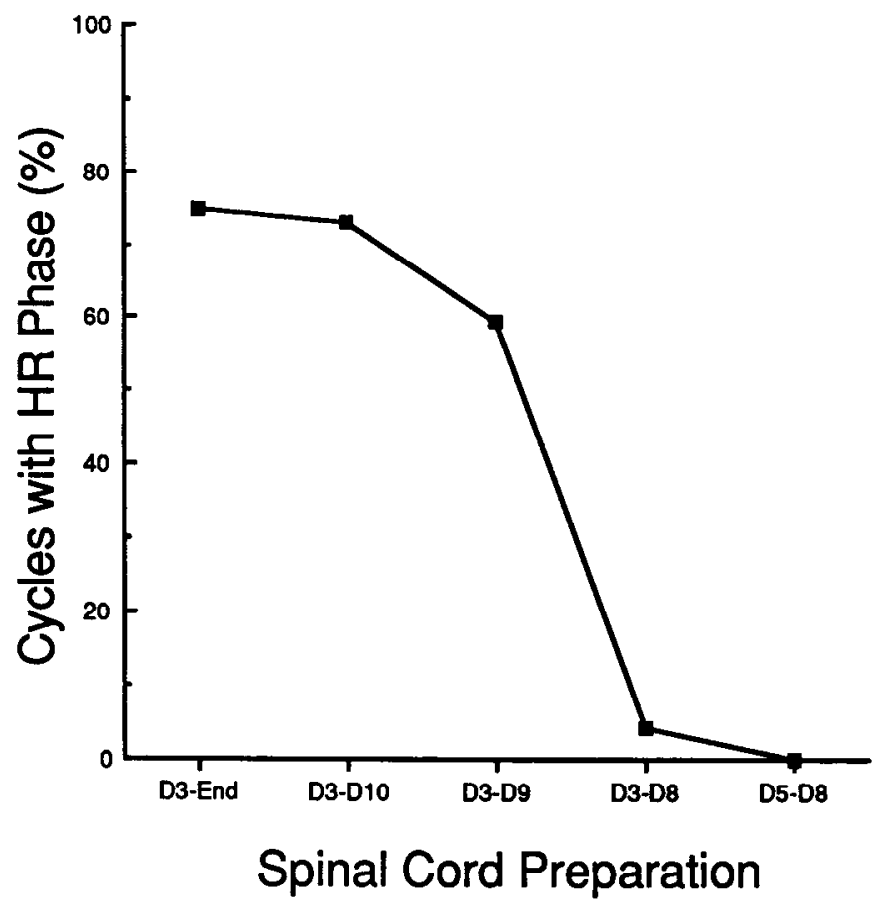

Figure 2. Plot showing the percentage of scratch cycles that exhibited a distinct hip retractor phase in 5 different spinal cord preparations in one turtle. Note that the percentage of cycles with hip retractor phases decreased to near zero with the loss of the posterior $80 \%$ of the hindlimb enlargement (D3-D8). After removal of segments D10-S2, which contain the motor pools for the hip retractor HR-KF, the hip retractor phase of the rostral scratch was monitored by recording from the dD8 nerve. 


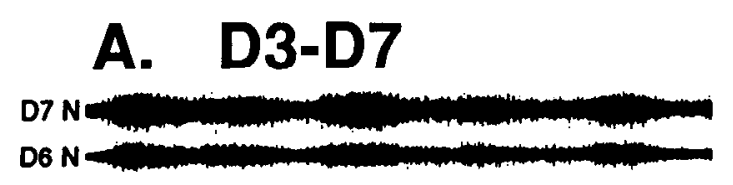

\section{D5-D9}

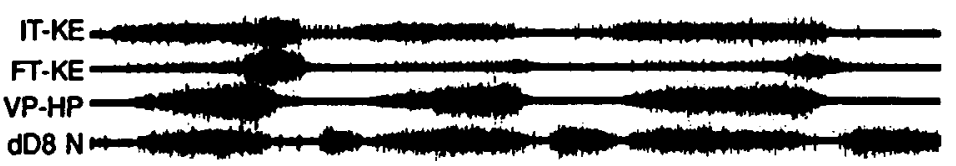

\section{E. D6-D8}

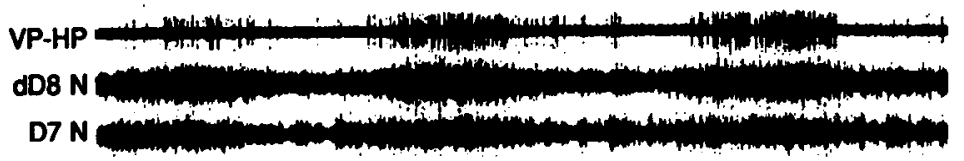

\section{B. D3-D6}
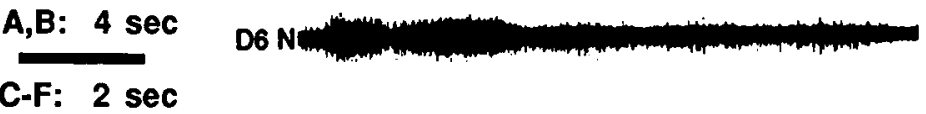

D. D6-D9
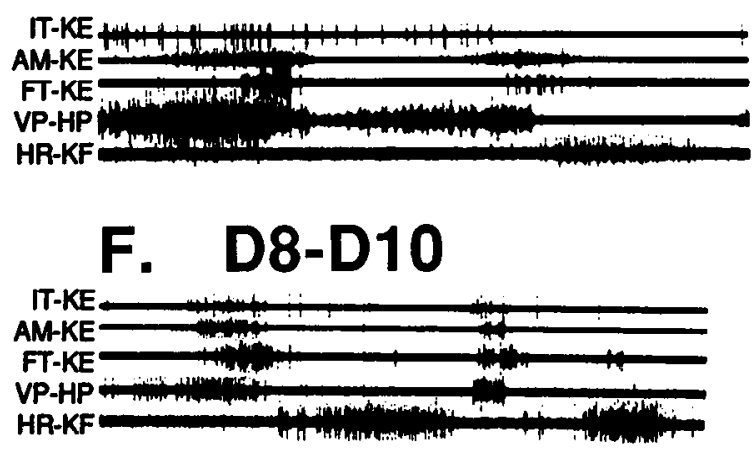

Figure 3. Rostral motor responses recorded in preparations consisting of 5 spinal cord segments or less. The stimulus positions used to elicit these motor responses: $A$, SP $2 ; B$, the midpoint of the inguinal plate; $C, \mathrm{M} 8.0 ; D$ and $E$, SP 1 . The response shown in $F$ was elicited by moving the hindlimb to a new position.

13 turtles with D3-D9 intact, 9 produced motor pattern synergies with rostral scratch characteristics. In 12 out of 12 turtles with D3-D10 intact, stimulation in the rostral receptive field produced motor patterns with uniquely rostral characteristics. Therefore, the posterior $40-80 \%$ of the hindlimb enlargement is not necessary for the production of the synergies of a rostral motor pattern.

If the posterior $40-80 \%$ of the enlargement is unnecessary for the production of a rostral motor pattern, then what happens when the entire enlargement is removed? Figure $3, A, B$, shows an experiment from one turtle designed to answer this by recording from motor pools outside of the enlargement. Figure $3 A$ shows 5 bursts of simultaneous activity in the D6 and D7 nerves in a preparation with segments D3-D7 intact; Figure $3 B$ shows 2 bursts of activity followed by weakly modulated activity in the D6 nerve in a preparation with segments D3-D6 intact. In controls, the D6 and D7 nerves were active mainly during the hip-protraction phase of the rostral scratch (data not shown). The motor responses shown in Figure $3, A, B$ (2 of the most vigorous seen in such preparations), indicate that rhythmogenesis can occur in response to rostral site stimulation in the absence of the hindlimb enlargement. With such limited monitors, it is not possible to determine if these spinal cord segments can produce motor pattern synergies that are specific to the rostral scratch form. However, some aspects of the rostral CPG must reside in segments anterior to segment D8. In 4 out of 4 turtles, a D3-D7 preparation produced distinct bursts of activity in response to stimulation within the rostral receptive field. In 3 out of 3 turtles, a D3-D6 preparation produced activity in the D6 nerve that showed rudimentary increases and decreases in a roughly rhythmical fashion. The rhythmogenic capabilities of the D3-D6 preparations were consistently weaker and more variable than those of the D3-D7 preparations, implying that key components of rostral scratch rhythmogenesis reside in segment D7.

What is the minimum length of spinal cord that can still produce a rostral motor pattern or basic rhythmicity? Figure $3 C$ shows a rostral motor pattern produced in a preparation consisting of segments D5-D9. The characteristic activation of FT-KE during the latter half of VP-HP activation and the occurrence of a hip-retractor phase (the second $\mathrm{dD} 8$ nerve burst here) after their offsets can be seen. Figure $3 D$ shows a 4 -segment preparation, D6-D9, that produced a motor response that displayed many uniquely rostral scratch characteristics. Two cycles of response are shown. The first cycle showed a deletion of HR$\mathrm{KF}$. The timing of FT-KE activation, during the latter half of VP-HP activation, is characteristic of a rostral scratch. Also characteristic of a rostral motor pattern, AM-KE began its activity before FT-KE, while these 2 nerves showed similar offset times. Thus, this preparation, containing just 4 segments, produced a response with many characteristics of the rostral motor pattern. In response to rostral receptive field stimulation, another 4-segment preparation, D5-D8, produced a motor pattern showing coactivation of VP-HP and the dD8 nerve (data not shown).

Figure 3, $E$, $F$, shows 2 different 3-segment preparations, D6D8 and D8-D10, respectively, that have only segment D8 in common. In response to rostral receptive field stimulation, the D6-D8 preparation produced 3 cycles of motor output that consisted of 3 bursts of coactivity in all 3 nerves (Fig. $3 E$ ). The D7 nerve recording showed some additional low-level activity that occurred during the brief quiescent periods of both VP-HP and the $\mathrm{dD} 8$ nerve; whether this activity represents a hip-retractor phase cannot be determined in this preparation, although this particular turtle did display low-level hip-retractor correlated activity in the D7 nerve in control preparations. Figure $3 F$ shows a unique response $(n-1)$. This D $8-\mathrm{D} 10$ preparation is separated from the rostral receptive field, the most posterior part of which is innervated by segment D6. This motor response was elicited accidentally while repositioning the immobilized hindlimb to achieve better access to the pocket receptive field. The motor response displayed several rostral scratch charac- 
teristics, in addition to some pocket scratch characteristics (Fig. $3 F$ ). In each of these 2 cycles, FT-KE was coactive with VP$\mathrm{HP}$, a characteristic that is specific to the rostral scratch form. The shorter VP-HP burst in the second cycle is more characteristic of the pocket motor pattern. This second cycle also had a small second FT-KE burst during HR-KF activity; the timing of this second burst is characteristic of the pocket scratch. Based upon these factors, the 2-cycle response resembles one cycle of rostral scratch followed by a second cycle showing a mixture of rostral and pocket features. Such mixed responses were seen in response to transition zone stimulation (Robertson et al., 1985) and to simultaneous stimulation of sites in 2 different receptive fields (Stein et al., 1986).

Preparations containing just segments D6-D7 produced weak bursts of activity in the D7 and D6 nerves in response to stimulation in the rostral scratch receptive field. Similarly, preparations containing segment D6 alone produced activity in the D6 nerve that showed small fluctuations in amplitude (data not shown). The rhythmicity in the D3-D7 and D3-D6 preparations illustrated in Figure $3, A$ and $B$, respectively, combined with these experiments showing a lack of large-amplitude rhythmicity in either a D6-D7 or a D6 preparation, implies that (1) there is only a very limited capacity for rostral rhythmogenesis anterior to the hindlimb enlargement, and (2) rostral rhythmogenesis anterior to the enlargement may require either more anterior segments or a length of spinal cord longer than 2 segments.

One feature of the rostral motor pattern that changed as segments were removed was cycle period. Figure $4 A$ shows a plot of cycle periods for one experiment. The D3-End preparation produced rostral scratch cycles with a mean period just under $3 \mathrm{sec}$. As more segments were removed, there was most often a gradual increase in the cycle period. The slight decrease in mean period in going from the D3-D10 to the D3-D9 preparation was due to the increase in the occurrences of hip-retractor phase deletions (see Fig. 2). Only 6\% (3/52) of the cycles in the D3-D10 preparation showed an absence of the hip-retractor phase; $51 \%(33 / 65)$ of the cycles in the D3-D9 preparation showed an absence of the hip-retractor phase. The average cycle periods for the D3-D10 and D3-D9 preparations were identical when we measured only those cycles that displayed a hip-retractor phase. The last 2 preparations were severely reduced in their rhythmogenic capabilities compared with preparations with enlargement segments intact. For the experiment illustrated in Figure 4 $A$, we tested the hypothesis that removal of segments resulted in rostral cycle periods greater than the control. Using a 1-tailed Mann-Whitney $U$ test, we confirmed this hypothesis at a significance level of $p<0.01$, except for the D3-D9 preparation, which was significantly greater than the control at a level of $p<0.03$ (Siegel, 1956). The more highly reduced preparations, containing segments D3-D8, D3-D7, and D3-D6, respectively, showed a marked decrease in the number of cycles produced for each episode of stimulation (data not shown). Whereas a control scratch episode would cycle for the duration of the stimulation, these reducecd preparations would produce episodes of 5 cycles or less.

\section{Pocket scratch generation}

The fictive pocket scratch motor pattcrn is characterized by the coactivation of FT-KE with HR-KF and by their antagonistic relationship with VP-HP (Fig. $5 A$ ). FT-KE activity can occur during the initial part of HR-KF activation (Fig. $5 A$ ) or run
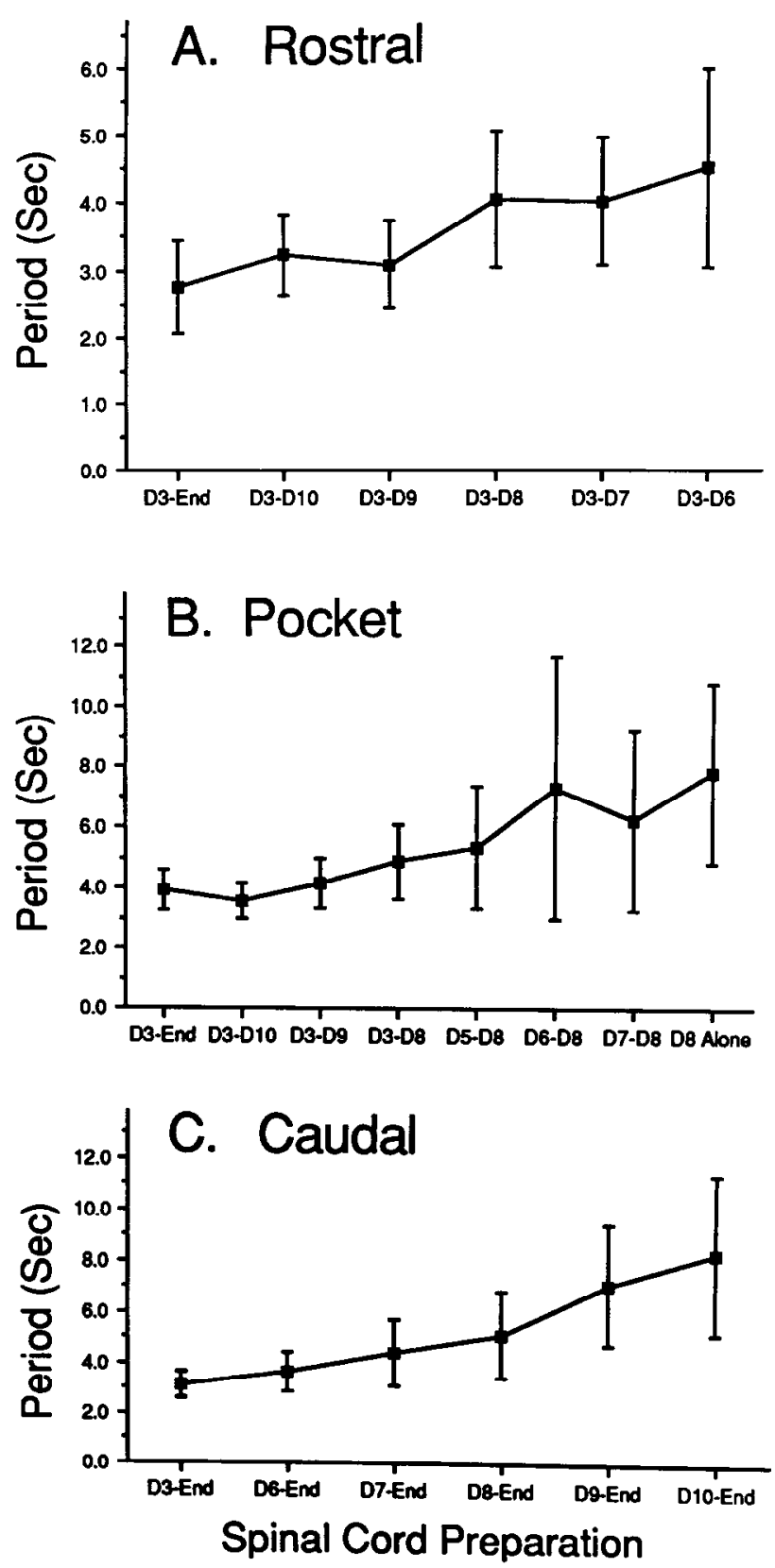

Figure 4. Plot of the mean cycle period of each preparation in one experiment for each scratch form: $A$, rostral; $B$, pocket; $C$, caudal. Error bars, SD of the mean. Note the steady increase in the mean cycle period and the concomitant increase in the variability of the cycle period as more segments are removed from each preparation. The number of cycles measured for each data point were as follows: $A, 30$ cycles or more; $B, 36$ cycles or more, except the D8 Alone preparation, which produced 12 cycles; $C, 63$ cycles or more.

throughout the length of the HR-KF phase (Fig. $6 B$; see also Robertson et al., 1985). FT-KE sometimes displays 2 bursts of activity in the fictive pocket scratch, one at the onset of HR$\mathrm{KF}$ activity and the other near HR-KF offset (data not shown). The pocket scratch is further characterized by the sequential timing of the onsets and offsets of IT-KE, AM-KE, and FT-KE, respectively (Fig. $5 A$ ). The AM-KE nerve begins its activity near the offset of IT-KE and continues its activation past the offset of VP-HP. This feature is different from the rostral scratch, 


\section{A. D3-End}

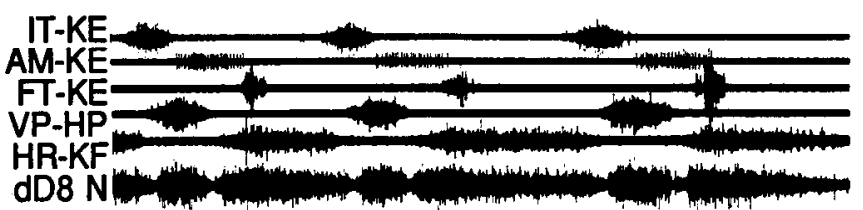

B. D3-D10

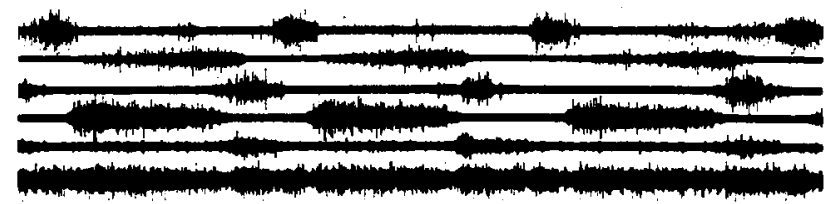

D. D3-D8

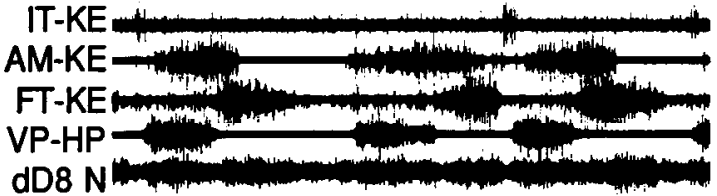

A-D: 2 sec
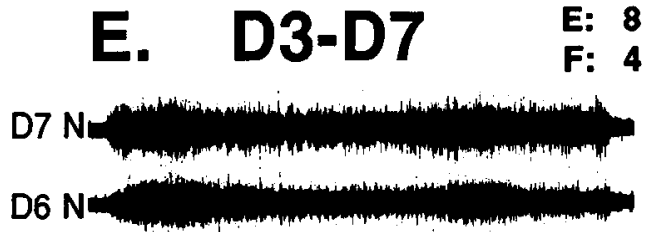

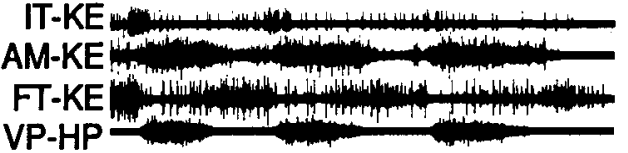

F. D3-D6

D6 $\mathrm{N}$

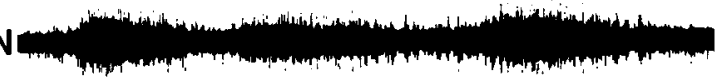

Figure 5. The posterior $40-80 \%$ of the hindlimb enlargement is not necessary for the production of a pocket scratch motor pattern. This figure is a composite of scratch episodes from several turtles. $A-D$, Each episode shows 3 cycles of a pocket motor pattern recorded in immobilized turtles and elicited by stimulation of sites in the pocket receptive field; $E, 2$ peaks of activity in response to stimulation in the pocket receptive field; $F$, 3 bursts of activity in response to stimulation of a site in the rostral-pocket transition zone (see Mortin et al., 1985). Note that the dD8 nerve recordings in $A-C$ showed 2 bursts of activity in the pocket scratch, one coactive with VP-HP and the second with HR-KF (there is no HR-KF monitor in $C$ ). The stimulus positions that elicited each scratch response: $A, \mathrm{~F} 4 ; B$ and $C, \mathrm{~F} 6 ; D, \mathrm{~F} 3.5 ; E, \mathrm{~F} 8$; and $F, \mathrm{~F} 1$.

where AM-KE turns off near the offset of VP-HP (cf. Figs. $5 A$ and $1 A$ ).

Figure 5, a composite of scratch episodes from several experiments, shows that the posterior 2-4 segments of the hindlimb enlargement are not necessary for the production of a pocket scratch motor pattern. Figure $5 A$ shows 3 cycles of a control pocket scratch. In the pocket scratch, the dD8 nerve showed 2 bursts of activity: the first coactive with VP-HP and the second coactive with HR-KF; these 2 bursts usually had similar amplitudes and thus were not easily distinguished from one another (Fig. $5 A$ ). The timing of the second dD 8 nerve burst correlated most closely with HR-KF activity but not with FTKE.

A D3-D 10 preparation produced a pocket motor pattern (Fig. $5 B$ ). A D3-D9 preparation produced a normal pocket motor pattern, except for 2 minor changes (Fig. $5 \mathrm{C}$ ). First, the loss of segment D10 usually resulted in the loss of the HR-KF motor pool, which resides in segments D10-S2. The dD8 nerve recording in Figure $5 C$, however, showed that there still was a hip-retractor correlated phase of activity in the motor response; this burst in the dD8 nerve was coactive with FT-KE, a characteristic unique to the pocket scratch. The second change seen at this stage was a reduction in the size and duration of the burst in IT-KE (cf. Fig. 5, $A, C$ ). The IT-KE motor pool is located largely in segments D8 and D9 (Ruigrok and Crowe, 1984); the reduction in IT-KE nerve activity that occurred with the loss of segment D10 cannot be explained by the loss of IT-KE motor neurons. Therefore, this reduction in the IT-KE burst implies that there was an alteration in the interneurons responsible for activating the IT-KE motor pool in the pocket scratch. A similar decrease in the activation of the IT-KE motor pool was not seen in the rostral scratch when D10 was removed (Fig. $3 C$ and control in Fig. 1A). Thus, a specific component of the pocket CPG, responsible for activation of the IT-KE motor pool, probably resides in segment D10; additional CPG elements involved in IT-KE activation reside in more anterior segments, as the remaining units were activated at the normal time. With the posterior 3 enlargement segments removed (Fig. 5C), the sequence of activation and inactivation of IT-KE, AM-KE, and FT-KE showed characteristics similar to the control pocket motor pattern.

A D3-D8 preparation produced a motor pattern that still displayed several characteristics specific to the pocket scratch (Fig. $5 D$ ). This preparation, with $80 \%$ of the enlargement removed, produced a rough alternation between the activity of VP-HP and FT-KE in this turtle. Most often, the FT-KE motor pool was lost with the removal of segment D9. In this example, FT-KE activity was still present. FT-KE had some activity throughout the response, but its frequency of activation increased greatly near the offset of VP-HP, and continued at this higher intensity throughout VP-HP quiescence. Additionally, the sequential timing of IT-KE, AM-KE, and FT-KE, was consistent with a pocket motor pattern (Fig. $5 D$ ).

Without the enlargement, the turtle displayed a significantly reduced ability to produce rhythmical motor outputs (Fig. 5, $E$, $F$ ). A D3-D7 preparation produced 2 slow peaks of modulated activity in response to stimulation within the pocket receptive field (Fig. 5E). The D7 and D6 nerves showed none of the 


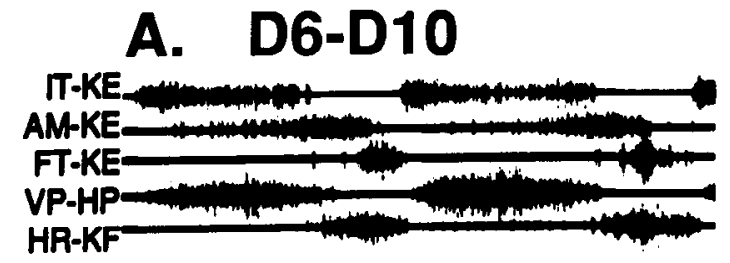

C. D6-D8

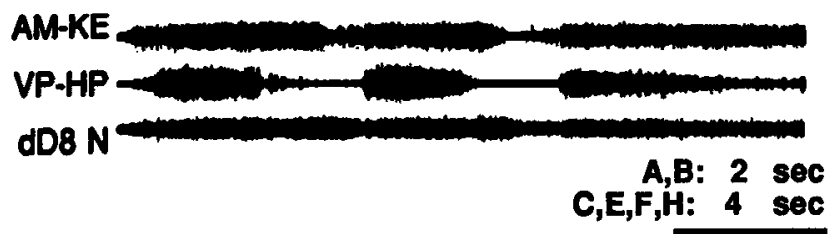

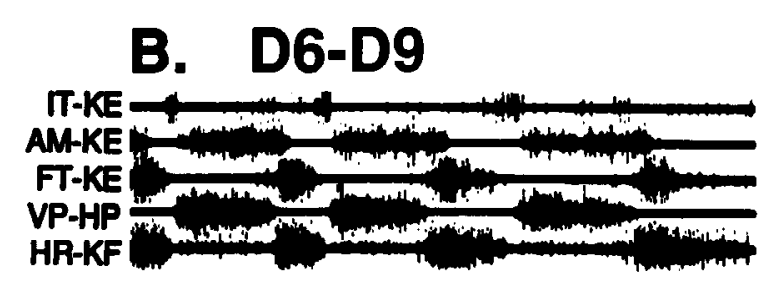

D. D8-D10

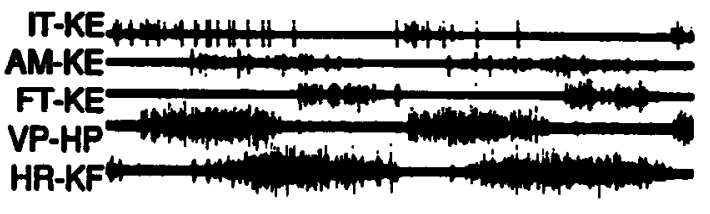

D: 1 sec G: 8 sec

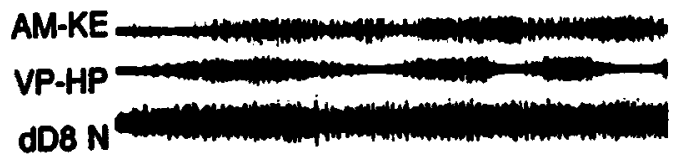

G. D7 Alone

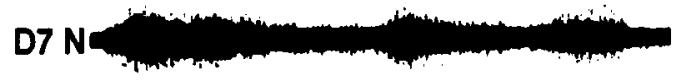

F. D8-D9

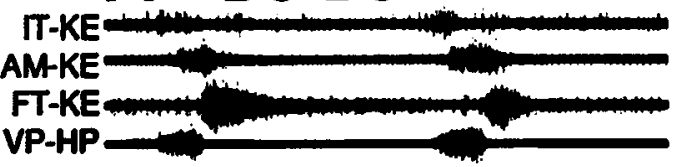

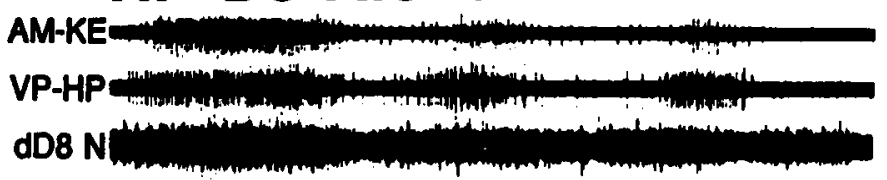

Figure 6. Motor patterns recorded in response to pocket site stimulation in preparations consisting of 5 spinal cord segments or less. Note that 2 different 3-segment preparations $(C$ and $D$ ) produced motor patterns with pocketlike characteristics, as did the 2-segment preparation in $F$. Two different 1 -segment preparations showed rhythmical motor outputs $(G$ and $H$ ). The stimulus positions used to elicit these responses: $A, C$, and $H$, $\mathrm{F} 8 ; B, E$, and $F, \mathrm{~F} 7 ; D, \mathrm{~F} 9$; and $G, \mathrm{~F} 2 . \mathrm{F} 2$ is at the posterior edge of the rostral-pocket transition zone.

discrete bursting and quiescence seen in other nerves in the previous preparations. A D3-D6 preparation displayed 3 bursts of activity in the D6 nerve in response to stimulation of a site in the rostral-pocket transition zone (Fig. $5 F$; see Robertson et al., 1985, for control transition zone responses). Whether this sensory stimulation activated the rostral or pocket generator or both cannot be determined by the D6 nerve recording. There are still some rhythmogenic capabilities within the spinal cord anterior to segment D7.

What is the minimal extent of spinal cord that is sufficient to produce a pocket scratch motor pattern? Figure 6 , a composite from 5 turtles, illustrates responses elicited in reduced preparations containing 5 segments or less. Figure $6, A$ and $B$ show pocket motor patterns produced in preparations consisting of 5 and 4 segments, respectively. Segments D3-D5 are not necessary for the production of a pocket motor pattern. Two different 3-segment preparations are illustrated in Figure 6, C, D, for comparison. The D6-D8 preparation in Figure $6 C$ produced 3 cycles of motor activity in response to pocket receptive field stimulation. AM-KE showed a pocket-specific timing, beginning near VP-HP onset and continuing past VP-HP offset (cf. Fig. 6, $B, C$ ). One change that was seen occasionally at this time was a decrease in the quiescent period of AM-KE. In the first cycle in Figure 6C, AM-KE activity never ended, but just decreased its frequency and amplitude prior to the onset of the next VP-
HP burst. The second cycle of this episode shows a normal pattern: AM-KE activity stopped near the middle of the phase of VP-HP quiescence. The D8-D10 preparation produced a pocket motor pattern largely indistinguishable from controls (Fig. 6D). There was a lot of overlap in the activities of VP-HP and $\mathrm{HR}-\mathrm{KF}$, but this was the case in control preparations for this turtle. All of the remaining pocket motor pattern characteristics were normal, except for a decrease in the intensity of IT-KE and AM-KE (cf. Fig. 6, $A, D$ ). Therefore, a 3-segment preparation, consisting of only the anterior $60 \%$ of the enlargement, can produce a pocket scratch motor pattern. A D7-D9 preparation also produced a response that had pocket-specific nerve synergies, but with some alterations in the robustness of the rhythmicity (data not shown). Thus, 3 different 3-segment preparations, D6-D8, D7-D9, and D8-D10, can produce a pocketlike motor pattern. Key elements of the pocket scratch CPG must reside in these segments.

Figure $6, E, F$, shows responses elicited by pocket site stimulation in 2 different 2-segment preparations. In a D7-D8 preparation VP-HP was active in bursts, with AM-KE and dD8 nerve activities overlapping and continuing past cessation of VP-HP activity (Fig. 6E). There was no longer any distinction in phases in the dD8 nerve recording based upon differences in amplitude. The overall response in Figure $6 E$, however, is similar to the response seen in the D6-D8 preparation (cf. Fig. 6, 
A. D3-End

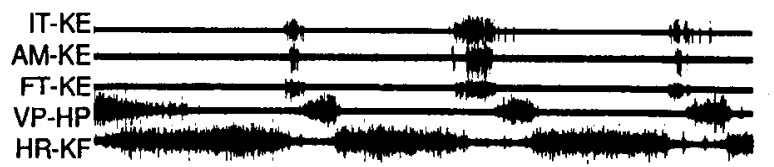

C. D10-End
B. D9-End

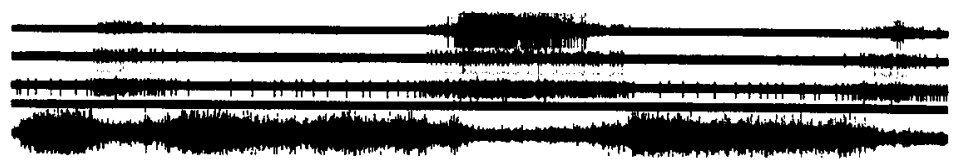

\section{S1-End}

HR-KF

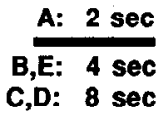

E. S2-End

\section{(1)}

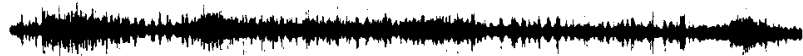

Figure 7. The posterior $80 \%$ of the hindlimb enlargement shows a reduced ability to produce a caudal scratch motor pattern. Each episode in this figure shows the motor response recorded in an immobilized turtle elicited by stimulation of sites in the caudal receptive field. $A-C$. Three cycles of response; $D$, roughly 2 slow and long bursts of activity in HR-KF, with a period of quiescence or decreased activity between these activity bursts; $E$, irregular modulations in HR-KF activity: this response was qualitatively different from those in any of the other 4 preparations. The stimulus positions that elicited each scratch response: $A-D, M 12.0$; and $E$, the ventral posterior midpoint.

$C, E$ ). A D8-D9 preparation produced 2 cycles that had characteristics specific for the pocket motor pattern (Fig. $6 F$ ). FT$\mathrm{KE}$ activity began near the offset of VP-HP activity. Also, the 3 knee extensors displayed the characteristic pocket pattern of staggered and sequential onsets and offsets. The timing of AM$\mathrm{KE}$ activation was specific to the pocket scratch, beginning after VP-HP onset and continuing past VP-HP offset. Thus, segments D8 and D9 contain key CPG elements sufficient for the production of a pocket motor pattern (Fig. $6 F$ ). This preparation, howcver, showed a reduced ability to display a rapid succession of cycles. Thus, segment D10 contains CPG elements that add to the robustness of the pocket motor pattern (cf. Fig. 6, $D, F$ ). A D6-D7 preparation displayed comodulation of weak activity in the D7 and D6 nerves (data not shown). These nerves were seen in control pocket experiments to display bursts of activity concurrent with VP-HP. There must be some rudimentary pocket rhythmogenic capabilities in D7 and D6.

Finally, Figure $6, G, H$, shows motor responses elicited in 2 different 1-segment preparations. With just segment D7, the D7 nerve, our only possible monitor, showed rhythmical bursts of activity followed by periods of silence (Fig. 6G). The robustness of the bursts was decreased with respect to controls, and the cycle period was increased. Still, there are rhythmogenic capabilities in some interneurons within segment $\mathrm{D} 7$. With just segment D8, stimulation within the pocket receptive field produced 3 cycles of concurrent bursts of activity in AM-KE, VPHP, and the dD8 nerve (Fig. $6 H$ ). The frequency of the activity in AM-KE and VP-HP was reduced compared with controls, but an organized and patterned response was still produced. The dD8 nerve was active throughout the response, but there was no distinct second peak of activity in the $\mathrm{dD} 8$ nerve per VPHP burst, and thus no clear hip-retractor correlated phase of activity. Similarly, AM-KE did not continue past VP-HP offset as it does in control pocket responses. However, key elements involved in pocket scratch rhythmogenesis must reside in segment D8.

Figure $4 B$ shows a plot of the mean cycle periods for a typical pocket scratch experiment. The control preparation, D3-End, produced a pocket scratch with a mean period of about 4 sec. The removal of the posterior 2 and even 3 segments of the enlargement did not appreciably alter the period of the response. The removal of D9 and then the anterior segments D3 and D4 produced an increase in the cycle period to about $5 \mathrm{sec}$. The SD of the mean also increased, as the length of each cycle during an episode and among episodes became more variable. Finally, the sequential loss of segments D5, D6, and D7, resulting in 3-, $2-$, and 1-segment preparations, respectively, produced a more dramatic increase in the cycle periods and in their SDs. In these highly reduced preparations, the mean cycle period had nearly doubled from control values. We tested the hypothesis that removal of segments resulted in pocket cycle periods greater than the control. Using a 1 -tailed Mann-Whitney $U$ test, we determined that the preparations containing segments D3-D8 or fewer segments produced cycle periods that were significantly greater than the control preparation ( $p<0.01$; Siegel, 1956). Thus, although the motor pattern continued to exhibit some pocketlike characteristics, there was a loss in the robustness of the motor output as measured by cycle period for these reduced preparations.

\section{Caudal scratch generation}

The fictive caudal scratch motor pattern is characterized by the activation of FT-KE betwecn the offset of HR-KF and the onset of VP-HP (Fig. $7 A$ ). The caudal scratch is also characterized by the nearly simultaneous onset and offset of all 3 knee extensors, IT-KE, AM-KE, and FT-KE, a feature that distinguishes the caudal scratch from the other 2 scratch forms. In addition, the activation of HR-KF usually is greater in amplitude and duration than the activation of VP-HP in the caudal scratch. The VP-HP burst displays a wide variation in intensity (amplitude and duration) in the caudal scratch.

Figure 7 demonstrates that key elements of the caudal scratch CPG reside anterior to segment $D 9$. The episodes illustrated in Figure 7 were obtained by stimulating sites in the caudal receptive field in 5 different spinal cord preparations from one turtle. 


\section{A. D3-End}
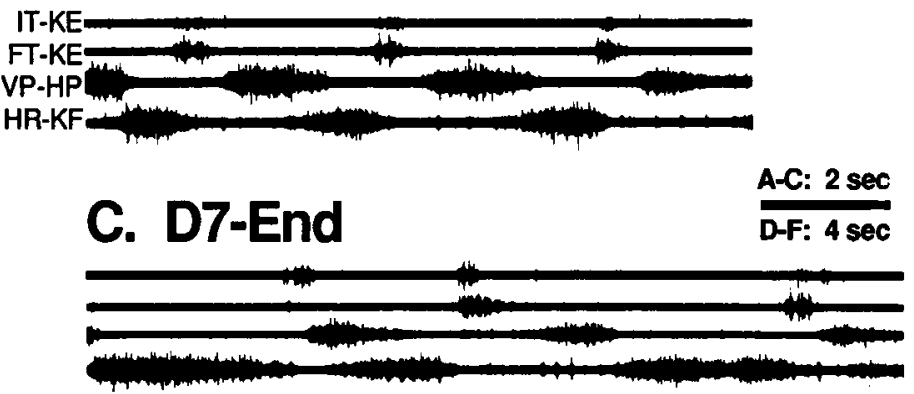

\section{E. D9-End}

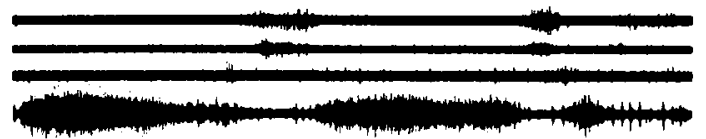

B. D6-End

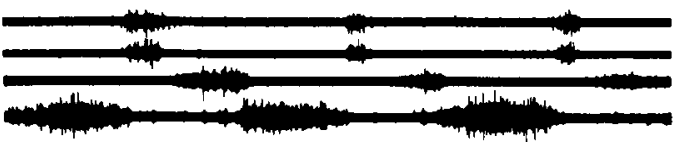

\section{D8-End}

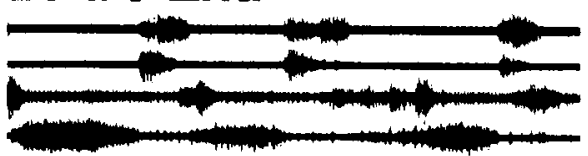

F. D10-End

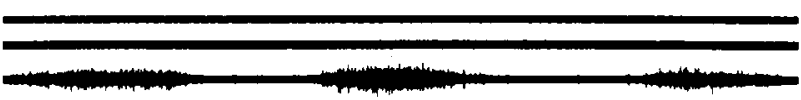

Figure 8. Segments D7 and D8 contain key elements of the caudal scratch CPG. Each episode of this figure shows 3 cycles of a caudal motor response from the same turtle. Note the characteristic activation of IT-KE and FT-KE near the offset of HR-KF activation, and the roughly alternating activation of VP-HP and HR-KF in $A-D$. The stimulus positions that elicited each scratch response: $A$ and $C, \mathrm{~A} 9 ; B, \mathrm{~A} 8 ; D$ and $F, \mathrm{~A} 5$; and $E, \mathrm{~A} 7$

Figure $7 A$ shows 3 cycles of a caudal scratch in a D3-End preparation. Figure $7 B$ illustrates the motor response elicited in a D9-End preparation. This preparation contained $80 \%$ of the hindlimb enlargement and all the caudal segments, including the innervation of the caudal receptive field. Several changes were seen in the caudal motor pattern at this stage. First, the cycle period increased significantly. A caudal scratch with a period of about $3 \mathrm{sec}$ (Fig. 7A) increased to more than $10 \mathrm{sec}$ (Fig. $7 B$ ). Second, there was no activity in VP-HP; tapping the dorsum of the foot in this preparation also elicited no activity in VP-HP, indicating a probable loss of the VP-HP motor pool. Other D9-End preparations showed this same absence of VPHP nerve activity (4/9), while the remainder showed some amount of VP-HP activity (5/9). The former turtles displayed a "prefixed" organization of the location of their motor pools within the spinal cord; the latter displayed a "postfixed" organization (Sherrington, 1892; Cruce, 1974; Mortin, 1987). Note, however, that the phase of the caudal scratch cycle where VPHP would normally be active, i.e., after the offset of FT-KE and before the onset of HR-KF, was absent in Figure $7 B$. The 3 knee extensors began their activities near the offset of HR-KF activity and remained coactive, as in control caudal scratches. If this were a normal caudal motor pattern, however, we would see a gap between the offset of the knee extensor nerve activites and the onset of the next HR-KF burst. One phase of the caudal motor pattern, therefore, was missing. The major effects in losing segments D3-D8 were (1) a large increase in the cycle period and (2) the loss of the exclusive hip-protractor phase of the cycle. The remaining synergies displayed by this preparation were similar to control caudal motor patterns.

The removal of segment $\mathrm{D} 9$ further increased the cycle period of the caudal scratch motor response (Fig. 7C). The activity in the 3 knee extensors, IT-KE, AM-KE, and FT-KE, was lost at this stagc; their motor pools usually arc locatcd in segments D8 and D9. The only monitor at this stage was HR-KF; in response to stimulation of a caudal receptive field site, there was a clear rhythmicity in HR-KF (Fig. 7C). This rhythmical activation and quiescence in HR-KF continued even after the removal of segment D10 (Fig. $7 D$ ). The motor response at this stage was a very slow and graded rhythmic increase and decrease in HR$\mathrm{KF}$ activity. Two slow bursts can be seen in Figure $7 D$, separated by a period of small-amplitude and low-frequency HR-KF activity.

The removal of segment $\mathrm{S} 1$ tended to disrupt any clear and regular on and off aspects of the activity in HR-KF (Fig. $7 E$ ). This preparation, S2-End, produced a motor output in HR-KF that was modulated in a highly irregular manner. Little, if anything, remained from the original caudal motor pattern. There were roughly 4 peaks in the amplitude of the HR-KF activity, with variable amplitude and frequency activity throughout the response (Fig. $7 E$ ). This example was the most rhythmical of all the responses elicited in this preparation. Therefore, segment S2 and the caudal segments $(\sim 16)$ contain very little of the caudal scratch $\mathrm{CPG}$, and produced at best only very rudimentary and highly irregular rhythms.

What segments, from D3 through D8, are responsible for the dramatic increase in the cycle period and for the loss of the exclusive hip-protractor phase that was seen in the second preparation of the previous figure? Figure 8 shows one experiment designed to answer this question. Figure $8 A$ shows 3 cycles of a caudal motor pattern. The coactivation of the 2 knee extensors shown here, IT-KE and FT-KE, occurred during the gap between the offset of HR-KF and the onset of VP-HP. Removing segments D3-D5 leaves a D6-End preparation and had no noticeable effect on the caudal motor pattern (Fig. $8 B$ ). The further removal of segment D6 produced only minor changes in the recorded motor patterns (Fig. $8 \mathrm{C}$ ). The motor response showed most of the characteristics of the control caudal pattern. There was no $\mathrm{FT}^{-} \mathrm{KE}$ activity in the first cycle of this 3-cycle response, and IT-KE showed a weak burst of activity in the last cycle; thesc variations were sometimes seen in control caudal scratch cycles, but they occurred more frequently in D7-End preparations. Thus, segments D3-D6 are not necessary for the production of a caudal scratch. Segment D6, however, may be 
involved in reinforcing certain aspects of the caudal motor pattern.

Figure $8 D$ shows a small, yet reliable change that generally occurred after the removal of segment D7, which leaves a D8End preparation. As shown in D, IT-KE began in the normal way, coactive with FT-KE; IT-KE activation, however, continued past the offset of FT-KE and partially overlapped the start of VP-HP. In addition, the duration of VP-HP activation became shorter and overlapped the first part of HR-KF. These minor changes were seen in other experiments, confirming that they are most likely effects duc to the loss of scgment D7. The D9-End preparation in this turtle showed an altered caudal motor output (Fig. $8 E$ ). As we saw in Figure $7 B$, the normal phase of VP-HP activity was absent as HR-KF activity commenced shortly after IT-KE and FT-KE activities ceased. In this turtle, however, some of the VP-HP motor pool was still intact, as tapping the dorsum of the foot to elicit a flexion reflex activated VP-HP motor neurons (data not shown). In this experiment, therefore, many of the interneurons responsible for activation of the VP-HP motor pool during the caudal scratch were located in segment D8. A few VP-HP units were activated in Figure $8 E$, but nothing like the robust bursts of activity seen in earlier preparations. Figure $8 E$ also shows that the cycle period of the motor response, which had been steadily increasing with each successive lesion, increased dramatically with the removal of segment D8. There was still, however, a coactivation of the 2 knee extensor nerves near the offset of HR-KF; this feature is specific to the caudal motor pattern. Thus, segment D8 contains key elements involved in the generation of the exclusive VP-HP phase in the caudal scratch and also helps maintain the periodicity of the motor pattern.

A D10-End preparation continued to show rhythmical bursts of HR-KF activity followed by quiescent periods; the other motor pools were lost at this stage (Fig. $8 F$ ). Whether any nerve activity occupied the phase of the rhythm when HR-KF was quiescent cannot be determined by this experiment. In another experiment, IT-KE activity alternated with HR-KF activity in a D10-End preparation (data not shown).

Figure $4 C$ shows a plot of the mean of the cycle period for the 6 preparations from the turtle shown in Figure 8 . In the control preparation, D3-End, stimulation of sites in the caudal receptive field produced scratch episodes consisting of 8 cycles or more, with a period of just over $3 \mathrm{sec}$. As segments were removed from the anterior end, there was a steady and gradual increase in the cycle period (Fig. $4 C$ ). The largest increase in the cycle period occurred when segment D8 was removed, even though the VP-HP phase of the cycle was lost. The mean period increased again after segment $D 9$ was removed. We tested the hypothesis that removal of segments resulted in caudal cycle periods grcater than the control. Using a 1-tailed Mann-Whitncy $U$ test, we determined that every reduced preparation in this experiment had a significantly greater cycle period than the control $(p<0.01)$. The cycle periods for each preparation were significantly greater than the cycle periods for the previous preparation at a significance level of $p<0.01$, except the final preparation, D10-End, which had a greater cycle period than the D9-End preparation at a significance level of $p<0.02$ (Siegel, 1956). Figure $4 C$ also shows that there was a steady increase in the variability of cycle periods as more segments were removed. Similar changes were described for the rostral and pocket scratch experiments. Caudal scratch episodes averaged around 8 cycles per stimulation sequence with segment D8 intact; removal of segment D8 resulted in scratch episodes that averaged just over 5 cycles. The additional removal of segment D9 resulted in even fewer cycles per episode; there was also less variability in the number of cycles per episode at this stage. Thus, segments D8 and D9 appear to contain key elements involved in the production of the caudal motor pattern.

\section{Discussion}

In the immobilized turtle, a preparation containing 3 spinal cord segments can produce pocket scratch motor output in response to tactile stimulation within the pocket scratch receptive field (Fig. 6D). These segments, D8-D10, comprise the anterior $60 \%$ of the hindlimb enlargement. This establishes that (1) these 3 segments contain sufficient neural circuitry to generate the synergies of the pocket scratch motor output and (2) key elements of the pocket scratch CPG are located in these segments. One spinal cord segment, either D7 or D8, can produce rhythmical bursting and quiescence in motor neurons in response to stimulation of sites in the pocket scratch receptive field (Fig. 6, $G, H$ ). Each of these 2 segments contains sufficient neuronal elements for motor rhythmogenesis. Similar results have been obtained for the generation of the swim rhythm in the lamprey: 2-4 spinal segments are sufficient to generate a rhythmic motor pattern (Cohen and Wallen, 1980; Grillner et al., 1982).

For each of the 3 forms of the scratch, key CPG elements reside in the anterior $60 \%$ of the hindlimb enlargement and in the segment rostral to the enlargement. The rhythmogenic and pattern-generating capabilities of the posterior $40 \%$ of the enlargement, as seen for caudal scratch generation, are extremely limited (see Fig. 7). These posterior enlargement segments, S1 and $\mathbf{S} 2$, are not necessary for the production of rostral and pocket scratches (Figs. $1 B$ and $5 B$, respectively). There is, therefore, an asymmetrical organization of CPG elements within and around the hindlimb enlargement; the anterior portion of the enlargement shows a greater capacity for producing the rhythmic synergies underlying each of the 3 scratch forms than the posterior portion of the enlargement.

A similar asymmetric organization has been described for the spinal circuitry underlying scratching in the cat (Berkinblit et al., 1978a, b; Deliagina et al., 1983). The hindlimb enlargement in the cat consists of the 5 segments L4-L7 and S1 (Romanes, 1964); these segments are homologous to spinal segments D8D10, S1, and S2 in the turtle (Ruigrok and Crowe, 1984). Immobilized, decerebrate cats produce a fictive scratch motor output in response to stimulation of either the pinna or upper cervical segments of the spinal cord (Deliagina et al., 1975, 1981). A complete transection between spinal segments L5 and L6 produced a preparation containing LS and more anterior segments; this preparation, without the posterior $60 \%$ of the hindlimb enlargement, generated rhythmical scratch-like activity in response to stimulation of segment C2 (Berkinblit et al., 1978a). The removal of 1 , and then 2 additional segments, L5 and $\mathrm{L} 4$, respectively, produced preparations that, in response to scratch stimulation, still generated rhythmic activity but with decreased vigor (Berkinblit et al., 1978a).

In a more extensive series of experiments utilizing localized cooling and selective lesions of different spinal cord regions, Deliagina et al. (1983) confirmed the conclusion that the anterior half of the hindlimb enlargement plays a crucial role in generating scratching in the cat. A preparation with L4 and more rostral segments intact produced stable scratch-like rhythms. In addition, segment L5, "isolated" by selective lesioning of the 
surrounding segments' gray matter, was capable of rhythmogenesis in response to pinna stimulation. The posterior half of the enlargement in the cat, segments L6, L7, and S1, generally was not capable of generating scratchlike rhythms; after a lesion of the gray matter of segments L4 and L5, continuous pinna stimulation produced only a couple of slow and wcak bursts of activity in more posterior segments after a prolonged delay $(>10$ sec; Deliagina et al., 1983). A similar dependence upon the anterior half of the hindlimb enlargement has also been demonstrated for cat stepping (Afelt et al., 1973; Grillner and Zangger, 1979). Further work with other limbed vertebrates is required to establish whether the asymmetric construction of the limb enlargement revealed in turtle and cat is a general feature of spinal cord organization.

Key elements of the CPGs for all 3 forms of the scratch in the turtle are located in segments D7-D10. The localization of key CPG elements for each scratch form within the same regions of the spinal cord is consistent with the hypothesis that there may be shared neuronal components among the scratch CPGs. One model of spinal cord interneuronal organization that postulates shared neuronal elements is the "unit burst generator" hypothesis (Grillner, 1981). This model is a modification of the half-center hypothesis, originally postulated by Brown and extended by Lundberg and Jankowska (Brown, 1911; also see Jankowska et al., 1967a, b; Lundberg, 1981). We have extended the Grillner hypothesis to describe a model for the CPGs responsible for the 3 forms of scratch in the turtle (Robertson et al., 1985). Both Grillner's and our hypotheses postulate a hip protraction (flexion) unit burst generator antagonistic to a hip retraction (extension) unit burst generator. In addition, these hypotheses postulate that the interneurons comprising each unit burst generator are active during the production of each of several forms of a task. We postulate that different forms of the scratch are produced by different synaptic linkages between these hip unit burst generators and a knee extension unit burst generator (Robertson et al., 1985; Stein et al., 1986). Direct recordings from the neurons responsible for the generation of motor patterns are now required to examine these hypotheses further.

Our work provides additional support for unit burst generator hypotheses by our demonstration that there is a differential localization of the interneurons that control hip protraction from those that control hip retraction. In the rostral scratch, the removal of segment D10, and then D9, produced a sequential decrease in the probability of occurrences of the hip retractor phase (Fig. 2). This suggests that interneurons controlling hip retraction are located mainly in segments D9 and D10. In these reduced preparations, the remaining segments generate normal timing characteristics, including robust bursts of hip protractor activity (e.g., Fig. 1, D, E). Thus, for the rostral scratch, interneurons controlling hip protraction are located anterior to segment D9, most likely in segments D7 and D8. In the pocket scratch, the 3 segment preparation D8-D10 produced a pocket motor pattern (Fig. $6 \mathrm{D}$ ). Interneurons controlling both hip protraction and hip retraction reside in these segments. Either segment D7 or D8 alone produced rhythmic bursts of activity in nerves synergistic to the hip protractor (Fig. 6, $G, H$ ). Interneurons controlling hip protraction, sufficient for the production of rhythmical bursts of activity, reside in segments D7 and D8. Our results with the pocket scratch are consistent with the notion that interneurons controlling hip retraction reside largely in segments D9 and D10. In the caudal scratch, the loss of segment
$D 8$, and to a lesser extent $D 7$, resulted in the loss of the exclusive hip protractor phase of the motor pattern (Figs. $7 B ; 8, D, E$ ). Again, this implicates segment $D 8$ as a site of interneurons controlling hip protraction, with some elements in segment D7. With a D10-End preparation, the turtle spinal cord still produccd rhythmic bursts in the hip retractor nerve (Figs. $7 C, 8 F$ ). This regular activation and inactivation of the hip retractor motor pool broke down as segments D10 and then S1 were removed (Fig. 7, D,E). These results implicate segment D10 (and possibly $\mathrm{S} 1)$ as containing interneurons controlling hip retraction in the caudal scratch. Thus, for the control of a given direction of hip movement, the same spinal cord segments are implicated for each of the 3 forms of the scratch: key elements of the hip protractor unit burst generator are located primarily in segments D7 and D8 and key elements of the hip retractor unit burst generator are located primarily in segments D9 and D10. Direct recordings from CPG interneurons within the turtle spinal cord are necessary to test these implications.

Our work is consistent with the hypothesis that some of the CPG interneurons for each scratch form are shared (Grillner, 1981; Robertson et al., 1985; Currie and Stein, 1988; Robertson and Stein, 1988). If CPG interneurons are shared, future work is required to determine whether the activation pattern of each shared CPG interneuron is different or the same for each form of the scratch. It is possible that there are CPG elements unique for each scratch form. Further work is required to locate these elements and to characterize how they interconnect with the shared CPG interneurons. In crustaceans, neuromodulators activate different linkages among CPG interneurons to generate different motor patterns (Marder, 1988). Further work with the turtle scratch may reveal whether neuromodulators play a role in producing diverse motor patterns in the vertebrate spinal cord.

\section{References}

Afelt, Z., N. B. Veber, and E. V. Maksimova (1973) Reflex Activity of Chronically Isolated Spinal Cord of the Cat, Nauka, Moscow.

Berkinblit, M. B., T. G. Deliagina, A. G. Feldman, I.M. Gelfand, and G. N. Orlovsky (1978a) Generation of scratching. I. Activity of spinal interneurons during scratching. J. Neurophysiol. 41:10401057.

Berkinblit, M. B., T. G. Deliagina, A. G. Feldman, I. M. Gelfand, and G. N. Orlovsky (1978b) Generation of scratching. II. Nonregular regimes of generation. J. Neurophysiol. 41: 1058-1069.

Bojanus, L. H. (1819) Anatome Testudinis Europaeae, Vilnae (reprint: Society for Study of Amphibians and Reptiles, 1970).

Brown, T. G. (1911) The intrinsic factors in the act of progression in the mammal. Proc. R. Soc. London [Biol.] 84: 308-319.

Cohen, A. H., and P. Wallen (1980) The ncuronal corrclate of locomotion in fish. "Fictive swimming" induced in an in vitro preparation of the lamprey spinal cord. Exp. Brain Res. 41: 11-18.

Cruce, W. L. R. (1974) The anatomical organization of hindlimb motoneurons in the lumbar spinal cord of the frog, Rana catesbiana. J. Comp. Neurol. 153: 59-76.

Currie, S. N., and P. S. G. Stein (1988) Electrical activation of the pocket scratch central pattern generator in the turtle. J. Neurophysiol. 60: 2122-2137.

Currie, S. N., and P. S. G. Stein (1989) Interruptions of fictive scratch motor rhythms by activation of cutaneous flexion reflex afferents in the turtle. J. Neurosci. 9: 488-496.

Deliagina, T. G., A. G. Feldman, I. M. Gelfand, and G. N. Orlovsky (1975) On the role of central program and afferent inflow in the control of scratching movements in the cat. Brain Res. 100:297-313.

Deliagina, T. G., G. N. Orlovsky, and C. Perret (1981) Efferent activity during fictitious scratch reflex in the cat. J. Neurophysiol. 45: 595604.

Deliagina, T. G., G. N. Orlovsky, and G. A. Pavlova (1983) The 
capacity for generation of rhythmic oscillations is distributed in the lumbosacral spinal cord of the cat. Exp. Brain Res. 53: 81-90.

Grillner, S. (1981) Control of locomotion in bipeds, tetrapods, and fish. In Handbook of Physiology, Sect. 1. The Nervous System, Vol. 2, Motor Control, V. B. Brooks, ed., pp. 1179-1236, American Physiological Society, Bethesda, MD.

Grillner, S., and P. Zangger (1979) On the central generation of 10comotion in the low spinal cat. Exp. Brain Res. 34: 241-261.

Grillner, S., A. McClellan, K. Sigvardt, P. Wallen, and T. Williams (1982) On the neural generation of "fictive locomotion" in a lower vertebrate nervous system, in vitro. In Brain Stem Control of Spinal Mechanisms (Fernstrom Foundation, Ser. No. 1), B. Sjolund and A. Bjorklund, eds., pp. 273-295, Elsevier Biomedical, Amsterdam.

Jankowska, E., M. G. M. Jukes, S. Lund, and A. Lundberg (1967a) The effect of DOPA on the spinal cord. 5. Reciprocal organization of pathways transmitting excitatory action to alpha motoneurones of flexors and extensors. Acta Physiol. Scand. 70: 369-388.

Jankowska, E., M. G. M. Jukes, S. Lund, and A. Lundberg (1967b) The effect of DOPA on the spinal cord. 6. Half-centre organization of interneurones transmitting effects from the flexor reflex afferents. Acta Physiol. Scand. 70: 389-402.

Kusuma, A., H. J. ten Donkelaar, and R. Nieuwenhuys (1979) Intrinsic organization of the spinal cord. In Biology of the Reptila. Vol. 10, C. Gans, R. G. Northcutt, and P. Ulinski, eds., pp. 59-109, Academic, New York.

Lundberg, A. (1981) Half-centres revisited. Adv. Physiol. Sci. 1: 155167.

Marder, E. (1988) Modulating a neuronal network. Nature 335: 296297.

Maxwell, J. H. (1979) Anesthesia and surgery. In Turtles: Perspective and Research, M. Harless and H. Morlock, eds., pp. 127-152, Wiley, New York.

Melby, E. C., Jr., and N. H. Altman (1974) Handbook of Laboratory Animal Science, Vol. 1. CRC, Cleveland.

Mortin, L. I. (1987) Mechanisms of Sensorimotor Integration in the Spinal Cord: Three Forms of Scratch Reflex in the Turtle. Ph.D. dissertation, Washington University, St. Louis, MO.

Mortin, L. I., and P. S. G. Stein (1985) Segmental properties of three forms of scratch reflex in the spinal turtle: Localization of input dermatomes and central pattern generating elements. Soc. Neurosci. Abstr. 11: 1021 .
Mortin, L. I., J. Keifer, and P. S. G. Stein (1985) Three forms of the scratch reflex in the spinal turtle: Movement analyses. J. Neurophysiol. 53: 1501-1516.

Robertson, G. A., and P. S. G. Stein (1988) Synaptic control of hindlimb motoneurones during three forms of the fictive scratch reflex in the turtle. J. Physiol. (Lond.) 404: 101-128.

Robertson, G. A., L. I. Mortin, J. Keifer, and P. S. G. Stein (1985) Three forms of the scratch reflex in the spinal turtle: Central generation of motor patterns. J. Neurophysiol. 53: 1517-1534.

Romanes, G. J. (1964) The motor pools of the spinal cord. In Progress in Brain Research. Vol. 2, Organization of the Spinal Cord, J. C. Eccles and J. P. Shade, eds., pp. 93-119, Elsevier, Amsterdam.

Ruigrok, T. J. H., and A. Crowe (1984) The organization of motoneurons in the turtle lumbar spinal cord. J. Comp. Neurol. 228: 2437.

Sherrington, C. S. (1892) Notes on the arrangement of some motor fibres in the lumbosacral plexus. J. Physiol. (Lond.) 13: 621-772.

Siegel, S. (1956) Nonparametric statistics for the behavioral sciences. McGraw-Hill, New York.

Stein, P. S. G. (1984) Central pattern generators in the spinal cord. In Handbook of the Spinal Cord, Vols. 2 and 3: Anatomy and Physiology, R. A. Davidoff, ed., pp. 647-672, Marcel Dekker, New York.

Stein, P. S. G. (1985) Neural control of the vertebrate limb: Multipartite pattern generators in the spinal cord. In Comparative Neurobiology: Modes of Communication in the Nervous System, M. J. Cohen and F. Strumwasser, eds., pp. 245-253, Wiley, New York.

Stein, P.S. G., and M. L. Grossman (1980) Central program for scratch reflex in turtle. J. Comp. Physiol. 140: 287-294.

Stein, P. S. G., G. A. Robertson, J. Keifer, M. L. Grossman, J. A. Berenbeim, and P. R. Lennard (1982) Motor neuron synaptic potentials during fictive scratch reflex in turtle. J. Comp. Physiol. 146: 401-409.

Stein, P. S. G., L. I. Mortin, and G. A. Robertson (1986) The forms of a task and their blends. In Neurobiology of Vertebrate Locomotion, S. Grillner, P. S. G. Stein, D. G. Stuart, H. Forssberg, R. M. Herman, and P. Wallen, eds., pp. 201-216, Macmillan, London.

Walker, W. F. (1973) The locomotor apparatus of testudines. In $B i$ ology of the Reptilla. Vol. 4, C. Gans and T. S. Parsons, eds., pp. 1100, Academic, New York. 